\title{
Effects of absolute luminance and luminance contrast on visual discrimination in low mesopic environments
}

\author{
Mathew Hunter ${ }^{1} \cdot$ Ben Godde $^{1} \cdot$ Bettina Olk $^{1,2}$
}

Published online: 14 October 2016

(C) The Psychonomic Society, Inc. 2016

\begin{abstract}
Recent research revealed considerable decline in visual perception under low luminance conditions. However, systematic studies on how visual performance is affected by absolute luminance and luminance contrast under low mesopic conditions $\left(<0.5 \mathrm{~cd} / \mathrm{m}^{2}\right)$ is lacking. We examined performance in a simple visual discrimination task under low mesopic luminance conditions in three experiments in which we systematically varied base luminance and luminance contrast between stimulus and background. We further manipulated eccentricity of the stimuli because of known rods and cones gradients along the retina. We identified a "deficiency window" for performance as measured by $d$ " when luminance was $<0.06 \mathrm{~cd} / \mathrm{m}^{2}$ and luminance contrast as measured by the luminance ratio between stimulus and background was below $<1.7$. We further calculated performance-based luminance as well as contrast efficiency functions for reaction times (RTs). These power functions demonstrate the contrast asymptote needed to decrease RTs and how such a decrease can be achieved given various combinations of absolute luminance and luminance contrast manipulations. Increased eccentricity resulted in slower RTs indicative of longer scan distances. Our data provide initial insights to performance-based efficiency functions in low
\end{abstract}

Electronic supplementary material The online version of this article (doi:10.3758/s13414-016-1219-z) contains supplementary material, which is available to authorized users.

Mathew Hunter

m.hunter@jacobs-university.de

1 Psychology \& Methods, Jacobs University Bremen, Campus Ring 1, 28759 Bremen, Germany

2 HSD Hochschule Döpfer, University of Applied Sciences, Cologne, Germany mesopic environments that are currently lacking and to the physical mechanisms being utilized for visual perception in these extreme environments.

Keywords Low luminance $\cdot$ Mesopic $\cdot$ Contrast efficiency function $\cdot$ Eccentricity $\cdot$ Visual discrimination

\section{Introduction}

Identification and discrimination of visual targets from distractors is one of the most common and complex tasks we are facing in our everyday lives. Despite the fact that our visual perceptual mechanisms are quite adept, we are still subject to limitations particularly evident in environments of extremely low luminance - specifically, the low mesopic luminance spectrum $\left(<0.5 \mathrm{~cd} / \mathrm{m}^{2}\right)$. Under such conditions, we face considerable changes and deficits in our perceptual abilities (i.e., brightness and colour perception - Purkinje shift) (Zele \& Cao, 2015). Naturally, this should extend to processes that are important for more complex tasks, such as efficient visual search processes. However, reviewing the literature, it is evident that even at processing stages that are important for visual search, such as the discrimination and identification of a stimulus, knowledge is lacking with respect to performance under low mesopic luminance. So far, almost all performancebased studies utilized simple detection tasks to indicate when a stimulus is perceived rather than how well a stimulus' perception (or the information obtained from the stimulus) influences behaviour, depending on varying properties, such as absolute luminance, contrast, and spatial frequency along the continuum from the mesopic to the scotopic luminance range. Data for low mesopic environments are missing, however. Walkey, Harlow, and Barbur (2006a, b) have provided one of the few studies touching on target discrimination 
or identification in a purely mesopic environment. The twophased study assessed: 1) isolated target detection and, 2) target identification within a search array, both as a function of luminance contrast and background luminance. Also, although studies completed in scotopic environments have demonstrated changes in luminance perception, it has not been investigated how these changes contribute to simple discrimination between two targets. Walkey et al. demonstrated that RTs decreased with increased contrast and reached asymptote at quickly when the target was viewed in photopic conditions $\left(<1 \mathrm{~cd} / \mathrm{m}^{2}\right)$. When considering the lowest background luminance $\left(0.01 \mathrm{~cd} / \mathrm{m}^{2}\right)$, RTs were slower and the range until reaching asymptote was longer with increasing contrast levels. While these findings hint to basic perceptual limitations observed in scotopic environments with varying contrasts, they are limited to one visual distance $\left(10^{\circ}\right)$ and did not include manipulations of eccentricity (cf. below).

Zele and Cao (2015) recently reviewed research on visual processing from primary retinal reception through tertiary cortical processing and ultimately behavioural perception at different illumination states from the scotopic to mesopic ranges. They concluded that, despite extensive research on the contributions of different retinal pathways within this transition, there is still a need for "performance-based luminance efficiency functions" for the mesopic spectrum, specifically, how performance is affected under these conditions and which changes of which variables affect performance. These functions would provide additional information with many industrial implications where visual search has to be completed under low ambient light conditions. The goal of the current study was to answer to the question of how low mesopic visual processing influences subsequent behavioural performance in a simple stimulus discrimination task with a novel behavioural efficiency function analysis. We specifically addressed target discrimination performance, as reflected by accuracy and reaction time (RT), as a function of absolute luminance, luminance contrast, and stimulus eccentricity within the low mesopic range. We used a simple visual discrimination task that required the identification of a target stimulus among two stimuli (target and distractor), which were presented at equidistance from the center at one of three predefined eccentricities. For this initial study, we decided for a small array to keep complexity at a minimum.

We will briefly review the literature regarding the effects of absolute luminance, luminance contrast, and stimulus eccentricity on performance in order to illustrate why we chose those variables for our study.

\section{Absolute luminance and luminance contrast}

When considering the role of luminance in visual perception independent of spectral wavelength, the primary properties investigated to date are absolute luminance of the targets or stimulus array and luminance contrast between targets and distractors or other stimuli. Inferences of the influence of luminance on these perceptions are based on mixed results regarding visual performance utilizing stimuli target isoluminance (no difference in absolute luminance value between target and distractor stimuli) as a metric. Isoluminant search paradigms typically reveal decreases in search rates (stimuli $/ \mathrm{ms}$ ) if targets and distractors approach absolute isoluminance (Skottun \& Skoyles, 2008). Alternatively, studies also have incorporated paradigms that estimated the required deviation/change from absolute isoluminance for a target to equal performance (delayed or increased RT) values observed for a target relative to distractors (called isoluminance novelty capture), and it is usually reported as a ratio value (the luminance of the target stimulus versus the background luminance). Although there are some inconsistencies in ratio values required for luminance to have similar properties of a target stimulus immersed within distractors (1:3, Rauschenberger (2003); 1:1.9 Watson and Humphreys (2002); 1:3 Kunar \& Watson, 2011), the consensus is that the ratio of change and not the absolute luminance difference is critical to influence performance. What must be borne in mind is that all these studies were conducted in optimal luminance environments (ratios have been tested in a range from 0.4 to $\sim 70 \mathrm{~cd} / \mathrm{m}^{2}$ ). Despite claiming to isolate magnocellular pathways, the luminance range is too broad (especially $>1 \mathrm{~cd} / \mathrm{m}^{2}$ ) incorporating parvocellular spectral ranges, which may confound assumptions about the parvocellular contribution to the identification or search process (Skottun, 2013) or vice versa. Also, and probably most importantly, it is recognized that true isoluminance may never be fully established in the lab setting (Braithwaite, Watson, Andrews, \& Humphreys, 2010; Skottun, 2013), which makes comparisons between studies difficult. Furthermore, the contribution of absolute luminance or luminance contrast may be more crucial under low mesopic environments due to the difference in the inherent perceptual responsiveness of the main visual pathways within this range.

\section{Stimulus eccentricity}

Many aspects of vision decline with increasing eccentricity, ranging from basic processes (e.g., acuity; Riggs, 1965) to higher order processes (e.g., word recognition; Lee, Legge, \& Ortiz, 2003) observed within the photopic ranges. Decline in some of these processes can be attenuated by increased stimulus size, but this effect does not hold true for all. When considering the low mesopic spectrum, luminance perception also varies with increasing stimulus eccentricity (Blackwell 1946, and may also be test size-dependent (Gloriani et al., 2016); however, in some instances perceptual abilities can sharpen at increasingly eccentric targets due to long wavelength preferences of the rods (Wald, 1945; Anstis, 2002). Such effects are attributed to the functional Purkinje shift as 
the rod density is minimal around the fovea and increases towards the periphery of the retina. From an evolutionary perspective, this is not surprising given the availability of long wavelength versus short wavelength light in naturally low luminance environments (i.e., night time). A key concept missing is the extrapolation from study results about pure sensitivity at low luminance conditions to perceptual capabilities, such as stimulus discrimination similar to the higherorder processes observed in photopic environments.

The concept of eccentricity affecting behavioural performance in the low mesopic spectral range has been primarily investigated using night time driving simulations (Plainis \& Murray, 2000, 2005; Plainis, Murray, \& Charman, 2005); however, these studies (as most in this range) are reaction time studies in a virtual simulator with no extrapolation to the subsequent performance that results from these perceptual limitations. Some studies have provided insight to changes in more complex driving behaviours (Alferdinck, 2006; Akashi \& Rea, 2002; Szalmas, Bodrogi, \& Sik-Lanyi, 2006); however, these represent tasks where the response is reflexive evasion to a light probe with no effort at discrimination of the task stimulus.

The present experiment was designed to fill the gap in research by using a simple two-alternative (absent/present), forced-choice reaction time task with two stimuli: a target and a distractor or two distractors. This allowed us to minimize the degrees of freedom in the task and only focus on the impact of luminance contrast and eccentricity under low mesopic luminance conditions. More specifically, we examined the effects of luminance contrast and absolute luminance changes with varying stimulus eccentricity in a low mesopic environment. In addition to RTs, we assessed signal detection accuracy $\left(d^{\prime}\right)$ under these conditions.

Due to the fact that rods should primarily be stimulated under these low mesopic conditions, we hypothesized that RT should be significantly faster to lateral stimuli than to more centrally located stimuli. However, we did not necessarily expect significantly better detection rates (increased $d$ ' scores) as a function of eccentricity as the task at surface value was not cognitively demanding. We also explored whether the effects would be specific to either manipulations of absolute luminance or luminance contrast independently or would be an interaction of the two. We expected that performance should decrease (slower RT and less accuracy) with lower ambient luminance values and decreased stimulus contrast consistent with other RT-based tasks.

With respect to the need for defined performance efficiency functions as raised by Zele and Cao (2015), we incorporated a simple, yet novel, way to calculate such a performance-based luminance efficiency function in the low mesopic spectrum. Based on the above-mentioned experiments, we decided to focus on the influence that the interaction of base luminance and stimuli contrast has on our task. This was completed assessing the contribution of the contrast provided by our stimuli within the task and its variation over different low mesopic base luminance values.

\section{Material and methods}

\section{Participants}

Nine participants ( 4 males, 5 females, age 18-20 years) took part and signed a written consent form. All had normal or corrected to normal vision. Participants were compensated with course credits or monetary remuneration. The experiment was conducted in accordance with the ethical standards laid down in the 1964 Declaration of Helsinki.

\section{Software and Hardware}

Experiment Builder ${ }^{\odot}$ (SR Research) was utilized for presentation of the displays and data recording. An IBM computer (ASI0 video card), and a 21" CRT-Monitor (ViewSonic G220F) were used to display the stimuli. All participants sat with the eyes at a distance of $57 \mathrm{~cm}$ from the monitor and placed their chin on a chin rest.

\section{Stimuli and Procedure}

Participants were brought into a darkened room where they received the instructions and were prepared for the required task. This served as the dark adaptation period for the study and lasted approximately 20 minutes. Most studies investigating perception in low mesopic environments typically ensure dark adaptation of the rods. While some researchers use a conservative adaptation period of 30 minutes (Yoshimoto, Okajima, \& Takeuchi, 2016), others measuring mesopic sensitivity as a qualifying measure reveal that only 5 minutes are sufficient to allow dark adaptation (Perez, Puell, Sanchez, \& Langa, 2003; Hertenstein, Bach, Gross, \& Beisse, 2016). The participants in this study completed approximately 20 minutes of dark adaptation, which should be sufficient for our study parameters (Eloholma, Ketomäki, Orreveteläinen, \& Halonen, 2006; Hiraoka, Hoshi, Okamoto, Okamoto, \& Oshika, 2015; Koefoed, Baste, Roumes, \& Hovding, 2015).

Participants were required to judge whether a target stimulus was present or absent in an array of two stimuli and responded by using the up (present) and down (absent) arrow keys on a standard keyboard with the right index finger for their response. In $50 \%$ of the trials, one target and one distractor stimulus were present, whereas two distractor stimuli were shown in the other $50 \%$ of the trials. The $50 \%$ probability that a target was present was made aware to the participants. Participants received no feedback on their response to prevent learning during the experiment. Each trial began with the presentation of a fixation cross $\left(1^{\circ}\right.$ visual angle) at the center of the display for $100 \mathrm{~ms}$ followed 
by a prestimulus interval of $800-1,200 \mathrm{~ms}$ (randomly generated) and the subsequent presentation of the array. At the beginning of a trial, participants had to fixate on the central fixation cross. In the course of the trial, they were allowed to fixate on the stimuli. A trial ended on participants' response or after $2000 \mathrm{~ms}$, whichever came first. RT and accuracy were measured and $d^{\prime}$ was calculated $\left(d^{\prime}=Z\right.$ (hit rate) $-Z$ (false-alarm rate); MacMillan and Creelman (2005)). $d^{\prime}$ was used to account for the falsealarm rates provided by the participants as the ambient lighting is extremely low, which may lead to a higher probability of participants to favour the target absent response.

Targets and distractors were circles with outside diameters of $0.5^{\circ}$ and superimposed concentric circles of $0.25^{\circ}$. Both the target and distractor stimuli were implemented as grayscale images. The target always had a higher luminance background (perceived darker center), whereas the distractors had a darker background (perceived brighter center). Examples are provided in Fig. 1.

The absolute luminance of the stimuli was controlled through manipulation of the RGB values reflecting its associated luminance. The associated stimuli luminance was determined through a previously calculated quadratic RGB/luminance curve derived from multiple lux measurements with a Gossen $®$ 5032B lux meter. ${ }^{1}$ Four base RGB values were selected, which defined the inner ring of the target and simultaneously the outer ring of the distractor annulus, $\mathrm{R}=\mathrm{x}, \mathrm{G}=\mathrm{x}, \mathrm{B}=\mathrm{x}$, where $\mathrm{x}=2,3,9$, or 13 and reflected the following absolute luminance values, respectively: $0.0329,0.0500,0.167,0.262 \mathrm{~cd} / \mathrm{m}^{2}$. All values were well below $0.6 \mathrm{~cd} / \mathrm{m}^{2}$, which is the estimated rod-cone discontinuity threshold (Rea \& Bullough, 2007). All R, G, and B values were equal, which eliminated colour perception of the targets creating grayscale perception. For clarity, the conditions will be identified with their corresponding RGB values throughout the paper (i.e., B2, B3, B9, B13 with $\mathrm{B}=$ Base luminance of the target or distractor) (Table 1).

Three levels of contrast difference between the inner and outer circles of the target were incorporated to assess the contribution of contrast at low luminance levels. Due to the nonlinear slope equation for the RGB luminance value (Table 1), contrast ratios were calculated to standardize the degree of change between the inside and outside circles of the target as opposed to simply adding linear incremental RGB units. For example, a linear addition of incremental units for the base value 2 , i.e., 3,4 and 5 , would not be an equivalent change in luminance $\left(\mathrm{cd} / \mathrm{m}^{2}\right)$ to the base value of 9 contrasted with values of 10,11 , and 12 . To account for this factor, each base value and its corresponding luminance had all proximal RGB luminance ratios calculated. For example, the ratios for base RGB value 2 were calculated against all absolute luminance values greater than its own value. Modulating the base RGB values by increments of one resulted in luminance-contrast

\footnotetext{
${ }^{1}$ Quadratic equation can be observed in Figure S1 placed in the supplement.
}

ratios within the range of 1.3-1.9 with a mean ratio value of approximately 1.7 (Table 1 ), which is within the ranges (1.31.9) reported earlier. Assignment of ratio difference as opposed to absolute luminance value is consistent with methodology of similar literature within the field (Rauschenberger, 2003; Watson \& Humphreys, 2002). Contrast ratio values were then assigned to one of three intervals defined by values below 1.7 (Low contrast), closest to 1.7 (Medium contrast), and greater than 1.7 (High contrast). The actual values for each base value and overall mean can be seen in Table 1 . Base luminance and contrast ratio were equivalent between the two stimuli for all trials.

The distance between the two stimuli was manipulated to assess the impact of eccentricity. Three different distances were selected $\left(1^{\circ}, 6^{\circ}, 10^{\circ}\right.$ of visual angle), referred to as inner, middle and outer, respectively. These distances were chosen to adequately disperse the retinal stimulation for inference of a potential lateralization preference or sensitivity of the rods and cones (Hadjikhani \& Toottell, 2000). At the same time, these values balance compromises between sufficient sampling of visual space, size of the display, and number of trials obtained. The probability that the target appeared on the left or right side was equal and the presentation order was randomized. See Fig. 1 for graphic representation of the stimuli and temporal timeline used for the experiment. The experiment consisted of six repetitions of all possible 72 conditions (4 Base (B2, B3, B9, B13) x 3 Contrast (low, medium, high) $\times 3$ Stimulus Distance (inner, middle, outer) x 2 Target Presence (absent, present), equaling 432 trials. Additionally, the participants were allotted 10 practice trials.

\section{Data analysis}

All experimental conditions were analyzed for both $d$ ' and RT (ms). Base luminance (B2, B3, B9, and B13) and luminance Contrast (low, medium, high) were used as independent factors. A third factor represented Eccentricity (inner, middle, outer) measured as stimulus distance. Only trials that had a valid response (RT between 300 and 2,000 ms) independent of accuracy were utilized for $d$ ' analysis, whereas only correct trials within the same response time frame were considered for the RT analyses. An additional factor Presence (absent, present) was introduced for RT analysis. ${ }^{2}$ Means were calculated for each condition and entered into repeated measures analysis of variance (ANOVA) and paired $t$ tests for all post hoc analyses. For all significant analyses violating assumptions of sphericity, Greenhouse Geisser corrections to the degrees of freedom were made when necessary. Only significant differences are reported in the results section.

\footnotetext{
${ }^{2}$ Because $d$ ' accounts for target false-alarm rate on absent trials, it is impossible to have a factor Presence.
} 


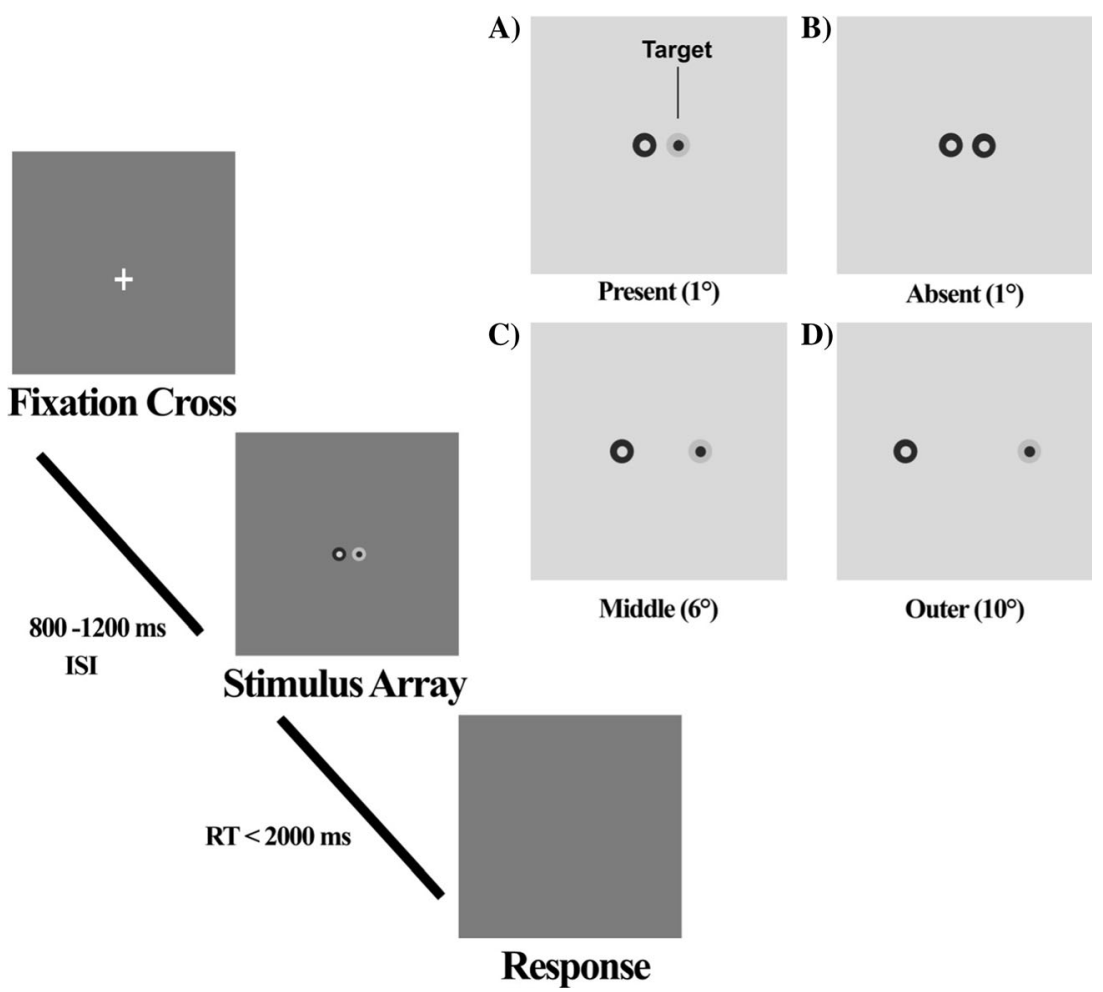

Fig. 1 Graphic representation of the temporal timeline. Each trial began with a fixation cross, ISI 800-1200, the array presentation and response. Upon key press the screen cleared and moved to next trial. The upper right hand corner shows the physical characteristic of the search stimuli.

Arrays A and B represent an example of target present versus absent at the inner distance $\left(1^{\circ}\right.$ between stimuli). Arrays $\mathrm{C}$ and $\mathrm{D}$ represent the alternative distances (Middle $-6^{\circ}$, Outer $-10^{\circ}$ ) used while a target was present (target absent trials are not shown).

For the performance efficiency function, a linear qualifier or coefficient that assesses both absolute luminance changes and the contrast simultaneously was created. While it was simple to assign a luminance coefficient to the base luminance variable (i.e., $\mathrm{cd} / \mathrm{m}^{2}$ ), the contrast coefficient was not as simple. When examining the stimuli in our paradigm, it was determined that two types of contrast would contribute to the general target perception, specifically, the contrast that was a result of the luminance of the stimulus from the luminance of the background and the inherent contrast within the stimulus (the alternating or

Table 1 Contrast ratio and actual RGB values (in brackets) utilized to define ratio threshold approximations as represented by overall ratio means

\begin{tabular}{lllll}
\hline Base RGB & $\begin{array}{l}\text { Low } \\
(\text { RGB })\end{array}$ & $\begin{array}{l}\text { Medium } \\
(\text { RGB })\end{array}$ & $\begin{array}{l}\text { High } \\
(\text { RGB })\end{array}$ & $\begin{array}{l}\text { Mean ratio } \\
(\text { RGB })\end{array}$ \\
\hline 2 & $1.52(3)$ & $2.04(4)$ & $2.59(5)$ & 2.05 \\
3 & $1.35(4)$ & $1.71(5)$ & $2.07(6)$ & 1.71 \\
9 & $1.49(13)$ & $1.67(14)$ & $1.85(15)$ & 1.67 \\
13 & $1.51(18)$ & $1.63(19)$ & $1.75(20)$ & 1.63 \\
Mean ratio & 1.46 & 1.74 & 2.03 & 1.74 \\
Mean ratio* & 1.45 & 1.67 & 1.89 & 1.67 \\
\hline
\end{tabular}

The purpose of calculating the mean ratio was to show the distribution of the individual values relative to the mean for each contrast level.

*mean ratio is excluding the inflation of the base 2 RGB ratio

periodic transition the absolute luminance of the light/dark circles). We quantified the combined contrast through two separate calculations reflecting the innate gradient of the stimulus (Michelson Contrast; Michelson, 1927) and the contrast of the stimulus from the background (Weber's fraction; Peli, 1990).

Michelson's contrast value is an optimal measure to quantify the contrast provided by the luminance difference between the outer and inner circles. Michelson's contrast is utilized to assess a contrast gradient of a sinusoidal pattern and given the fact that the stimulus varies periodically moving laterally across the stimulus (distractor: light - dark - light; target: dark - light dark) it seemed like the most appropriate measure. The contrast $\left(C_{M}\right)$ value is calculated as the ratio of the difference between the maximum and minimum luminance to the total luminance of the gradient and belongs to a range of 0 to 1 (Eq. 1).

$C_{M}=\frac{L_{\max }-L_{\min }}{L_{\max }+L_{\min }}$

Weber's fraction $\left(C_{W}\right)$ reflects the contrast between a single stimulus and its surrounding background luminance (Eq. 2).

$C_{W}=\frac{\Delta L}{L}$

$\Delta L$ is the incremental change in luminance of the target relative to $L$, which is the background luminance. For this 
study $L$ was $=0.001 \mathrm{~cd} / \mathrm{m}^{2}$ avoiding zero assignment as the denominator and $\Delta L$ was calculated as the max luminance of the brightest luminance value for each respective condition. For target present conditions this was reflected by the outer annulus value and for the target absent conditions this was reflected by the inner annulus value.

Previous researchers only incorporated either $C_{M}$ or $C_{W}$. We assumed, however, the Mickelson's contrast and Weber's fraction should importantly and differently influence perception. Thus, we calculated the interaction term as the product of the two values for this experiment (Eq. 3).

$C_{M W}=C_{M} \times C_{W}$

The contrast coefficients $\left(C_{M}, C_{W}, C_{M W}\right)$ were then correlated against the performance means (RT and $d^{\prime}$ ) for each condition. This additional analysis provided a more quantitative perspective than the discrete ANOVA analysis and is a preliminary step to presenting generalizable "performance-based efficiency functions" as alluded to by Zele and Cao (2015). The contrast coefficients for each condition are presented in Table 2.

\section{Results}

\section{Detection Accuracy (d')}

A 4 Base (B2, B3, B9, B13) x 3 Contrast (low, medium, high) x 3 Eccentricity (inner, middle, outer) repeated measures ANOVA demonstrated main effects of Base $\left(F_{(3,24)}=3.98 p\right.$ $<0.02$ eta $\left.^{2}=0.31\right)$ and Contrast $\left(F_{(2,16)}=16.34 p<0.001\right.$ eta $^{2}$ $=0.64)$ as well as a Base $\mathrm{x}$ Contrast interaction $\left(F_{(6,48)}=8.54\right.$ $p<0.0001$ eta $\left.^{2}=0.49\right)$. No other significant effects were demonstrated. The results are illustrated in Fig. 2.

As can be seen in Fig. 2 and confirmed by post-hoc paired $t$ tests, the interaction was driven by poorer performance at low contrast at $\mathrm{B} 2(1.85 \pm 0.29$; mean and $\mathrm{SD})$ and $\mathrm{B} 3(1.85 \pm$

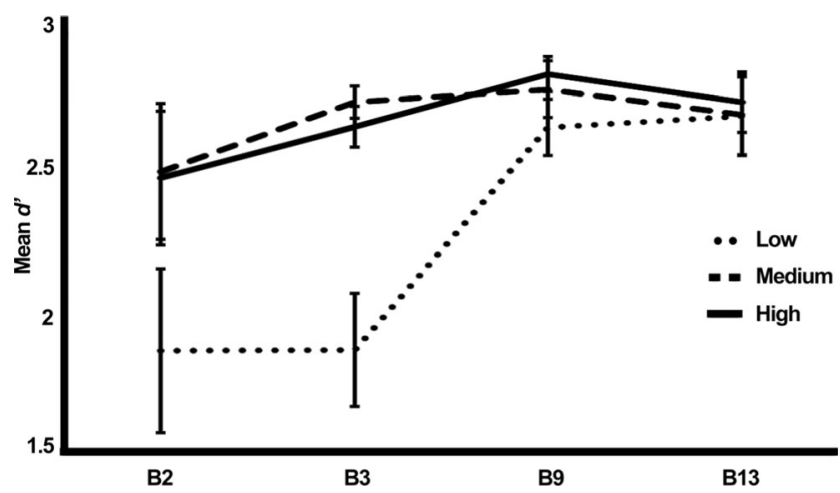

Fig. 2 Mean $d$ ' scores and SEMs for the base luminance and luminance ratio interaction. Base values are indicated by $\mathrm{B}$ character and its respective RGB value as described in the text. Contrast represents the following ratio conditions as described in the text: Low $\leq 1.7$, Medium $\sim 1.7$, High $>1.7$.

$0.20)$ base luminance levels $\left(<1.7 \mathrm{~cd} / \mathrm{m}^{2}\right)$ relative to the $\mathrm{B} 9$ $(\mathrm{B} 9=2.65 \pm 0.10)$ and $\mathrm{B} 13(\mathrm{~B} 13=2.69 \pm 0.14)$ levels $(\mathrm{B} 2<$ B9: $\mathrm{t}(9)=2.93 ; \mathrm{B} 2<\mathrm{B} 13: \mathrm{t}(9)=3.58 ; \mathrm{B} 3<\mathrm{B} 9: \mathrm{t}(9)=5.24$; $\mathrm{B} 3<\mathrm{B} 13: \mathrm{t}(9)=5.69 ; p<0.005$ for all these comparisons) with no significant differences between the B2 and B3 or B9 and $\mathrm{B} 13$. For the medium and high contrast conditions, there were no significant differences among the four base luminance levels (Medium: $\mathrm{B} 2=2.49 \pm 0.24, \mathrm{~B} 3=2.74 \pm 0.06$, $\mathrm{B} 9=2.79 \pm 0.10, \mathrm{~B} 13=2.70 \pm 0.15 ;$ High: $\mathrm{B} 2=2.47 \pm 0.24$, $\mathrm{B} 3=2.65 \pm 0.07, \mathrm{~B} 9=2.84 \pm 0.06, \mathrm{~B} 13=2.74 \pm 0.11$.

\section{Reaction Times}

Only accurate trials were selected for analyses with an overall mean of $91.5 \%(\mathrm{n}=9$, range $84 \%$ - $94 \%)$. A 4 Base (B2, B3, B9, B13) x 3 Contrast (low, medium, high) x 3 Eccentricity (inner, middle, outer) x 2 Presence (absent, present) repeated measures ANOVA revealed significant main effects of Base $\left(F_{(3,18)}=4.16 p<0.05\right.$ eta $\left.^{2}=0.45\right)$, Contrast $\left(F_{(2,12)}=15.20 p\right.$ $<0.01$ eta $\left.^{2}=0.75\right)$, and Eccentricity $\left(F_{(2,12)}=27.92 p<0.001\right.$

Table 2 Contrast coefficients represented as product of Michelson contrast and Weber's Fraction

\begin{tabular}{|c|c|c|c|c|c|c|c|c|c|c|c|c|}
\hline \multirow[b]{2}{*}{ Base } & \multicolumn{2}{|c|}{ Low $(<1.7)$} & \multirow[b]{2}{*}{$C_{M W}$} & \multicolumn{2}{|c|}{$\operatorname{Medium}(\sim 1.7)$} & \multirow[b]{2}{*}{$C_{M W}$} & \multicolumn{2}{|c|}{ High $(>1.7)$} & \multirow[b]{2}{*}{$C_{M W}$} & \multicolumn{2}{|c|}{ Mean } & \multirow[b]{2}{*}{$C_{\overline{M W}}$} \\
\hline & $C_{M}$ & $C_{W}$ & & $C_{M}$ & $C_{W}$ & & $C_{M}$ & $C_{W}$ & & $C_{\bar{M}}$ & $C_{\bar{W}}$ & \\
\hline 2 & 0.21 & 3.97 & 0.81 & 0.34 & 5.02 & 1.72 & 0.44 & 6.09 & 2.69 & 0.33 & 5.02 & 1.74 \\
\hline 3 & 0.15 & 6.03 & 0.90 & 0.26 & 7.11 & 1.85 & 0.35 & 8.20 & 2.86 & 0.25 & 7.11 & 1.87 \\
\hline 9 & 0.21 & 23.65 & 5.02 & 0.26 & 25.07 & 6.40 & 0.30 & 26.55 & 7.83 & 0.25 & 25.09 & 6.42 \\
\hline 13 & 0.20 & 36.54 & 7.46 & 0.24 & 38.24 & 9.13 & 0.27 & 40.01 & 10.86 & 0.24 & 38.26 & 9.15 \\
\hline Mean & 0.19 & 16.33 & 3.25 & 0.27 & 17.61 & 4.45 & 0.33 & 18.93 & 5.71 & 0.26 & 17.62 & 4.47 \\
\hline
\end{tabular}

$C_{M}=$ Michelson's contrast value

$C_{W}=$ Weber's fraction value

$C_{M W}=$ Contrast product

The purpose of calculating the mean ratio was to show the distribution of the individual values relative to the mean. 
$\left.\mathrm{eta}^{2}=0.85\right)$. The Base main effect demonstrated that the B2 and B3 base values were significantly slower than the B9 and $\mathrm{B} 13$ values, with no differences between the $\mathrm{B} 2$ and $\mathrm{B} 3$ and $\mathrm{B} 9$ and $\mathrm{B} 13(\mathrm{~B} 2<\mathrm{B} 3 \mathrm{t}=0.89 p=0.42, \mathrm{~B} 2<\mathrm{B} 9 \mathrm{t}=1.86 p=$ $0.06, \mathrm{~B} 2<\mathrm{B} 13 \mathrm{t}=2.37 p<0.05, \mathrm{~B} 3<\mathrm{B} 9 \mathrm{t}=3.41 p<0.05$, $\mathrm{B} 3<\mathrm{B} 13 \mathrm{t}=2.79, p<0.05, \mathrm{~B} 9<\mathrm{B} 13 \mathrm{t}=1.30 p=0.25)$. Within the Contrast main effect, the low contrast condition yielded significantly slower RTs than the medium and high contrast conditions (Low $>$ Medium $\mathrm{t}=4.97, p<0.01$; Low $>$ High $\mathrm{t}=3.45, p<0.05$; Medium $>$ High $\mathrm{t}=0.92, p=0.40)$ No differences were demonstrated between the Medium and High contrasts. The means and SEMs for both main effects are presented in Table 3.

The Eccentricity main effect reflected significantly slower RT in the middle and outer positions when compared against the inner position with no differences being observed between middle and outer positions. However, a Presence $\mathrm{x}$ Eccentricity interaction demonstrated that the significantly slower RTs for the middle and outer positions were evident only when the target was absent $\left(F_{(2,12)}=7.25 p<0.01\right.$ eta $^{2}=$ 0.59; Absent: Inner $>$ Middle $\mathrm{t}=4.37 p<0.01$, Inner $>$ Outer $\mathrm{t}$ $=6.99 p<0.001$, Middle $>$ Outer $\mathrm{t}=2.23 p=0.07$; Present: Inner $>$ Middle $\mathrm{t}=0.75 p=0.94$, Inner $>$ Outer $\mathrm{t}=-1.33, p=$ 0.24 , Middle $>$ Outer $\mathrm{t}=0.79, p=0.47$ ), while no differences were observed in the target present condition (Table $3 \mathrm{c}$ ).

\section{Luminance-based performance curves}

Association of performance with the condition contrast coefficients was analyzed for both $d$ ' and RT against the three coefficients with no yielding no significant results revealed for the individual coefficients $\left(C_{M}\right.$ and $\left.C_{W}\right)$; however, significant findings were demonstrated for the coefficient product $\left(C_{M W}\right)$. The

Table 3 Reaction time means and SEMs for Contrast (a), Base RGB (b) Value and Presence $x$ Eccentricity Interaction (c)

\begin{tabular}{lllllll}
\hline a) & Contrast & Mean & SEM & & & \\
& Low & 1027.93 & 58.88 & & & \\
Medium & 909.09 & 54.89 & & & \\
& High & 920.75 & 66.25 & & & \\
b) & Base* & Mean & SEM & & & \\
B2 & $1037.81^{*}$ & 91.06 & & & \\
B3 & 985.41 & 53.81 & & & \\
B9 & 905.37 & 54.28 & & & SEM \\
B13 & 881.48 & 57.97 & & & 63.70 \\
Absent & Mean & SEM & Present & Mean & \\
Inner & 879.27 & 63.93 & Inner & 930.81 & 62.49 \\
Middle & 988.38 & 62.85 & Middle & 932.85 & 63.77 \\
Outer & 1033.98 & 74.00 & Outer & 950.24 & 63.79 \\
\hline
\end{tabular}

*t test value between B2 > B9 was found to be approaching significance with a $p=0.06$, which is most likely a function of the increased SEM for the $\mathrm{B} 2$ condition.
12 coefficients (Base 4 (B2, B3, B9, B13) x Contrast 3 (Low, Medium, High) were fitted to the cumulative respective performance means first for a generalized function of the performance deficits. The greatest $r^{2}$ values for both measures $\left(r^{2}<\right.$ 0.40 ) were observed by fitting with power functions; however, the $\mathrm{B} 3$ medium contrast $\left(C_{M W}=1.85\right)$ presented visually as an outlier and was removed from the analysis. The remaining variables were then analyzed resulting in highly predictive power functions for both performance measures $\left(d^{\prime}(\mathrm{x})=\right.$ $2.05 \mathrm{x}^{0.15} \mathrm{r}^{2}=0.83$; RT $\left.(\mathrm{x})=1088.3 \mathrm{x}^{-0.09} \mathrm{r}^{2}=0.89\right)$. Scatterplots of the equations are presented in Figs. 3 and 4 respectively. Only analyses involving $C_{M W}$ produced significant results. Regression plots of the performance data with $C_{M}$ or $C_{W}$ resulted in $\mathrm{r}^{2}$ values $<0.10$ (data not shown).

This regression plot was further extrapolated across the Eccentricity $x$ Presence interaction observed for RTs. The results are demonstrated in Fig. 4 with equations and $r^{2}$ values. Similar to the previously identified equations $C_{M W}=1.85$ was removed from the analysis. The interaction effect of decreased RT for the innermost target position during the absent conditions which is evident in 5a; however, the purpose of this plot is to examine the disparity in $\mathrm{r}^{2}$ over the different conditions. $\mathrm{R}^{2}$ values indicate that at inner and outer eccentricities but not at the transition between the two positions performance fitted the power function well. The tightness of the power function was stronger in the target present than in target absence conditions.

\section{Discussion}

With this study, we investigated visual discrimination performance in low mesopic environments. We analyzed behavioural performance (RT and accuracy) in a simple stimulus discrimination task while also incorporating a novel behavioural efficiency function analysis. We specifically addressed discrimination performance as a function of absolute luminance, luminance contrast, and stimulus eccentricity within the low mesopic range.

We demonstrated that both signal detection accuracy and RT in a simple visual discrimination task faced considerable decreases in performance (smaller $d^{\prime}$ and increased RTs) when the absolute base luminance was below $<0.6 \mathrm{~cd} / \mathrm{m}^{2}$ and when the luminance contrast within the stimuli was below the defined 1.7 contrast ratio. The results are consistent with our initial hypotheses of decreased performance attributed to the decreases in absolute luminance and contrast ratios of the stimuli.

Interestingly, for RT the performance decrease was only present as main effects of base luminance and luminance contrast in the low contrast conditions, while decrease in $d$ ' was found to be a function of the interaction of the two factors. These results suggest that RTs may be more susceptible to performance decrease in only one luminance characteristic with no compounding effect of a performance decrease in 


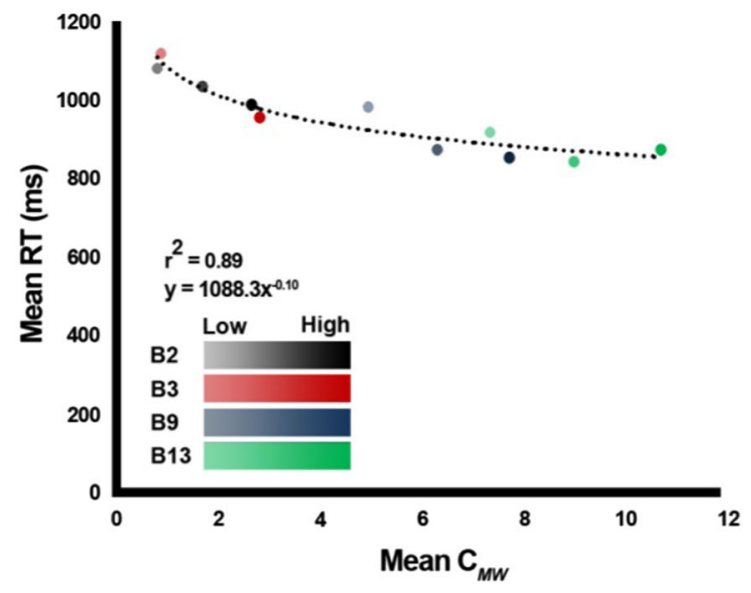

Fig. 3 RT and $d$ ' efficiency equations. Plot of performance measures against the contrast coefficient $\left(C_{M W}\right)$ for each condition. Data points are colour coded to represent both base luminance $($ Black $=\mathrm{B} 2$, Red $=$ $\mathrm{B} 3$, Blue $=\mathrm{B} 9$ and Green $=\mathrm{B} 13)$ and contrast $($ Light $=$ Low, $\mathrm{Mid}=$

the other luminance property. This is also supported by the increased $\mathrm{r}^{2}$ value in the RT regression equation. A change in either of the $C_{M}$ or $C_{W}$ values effectively changing the $C_{M W}$ will affect RT to a greater degree than $d$ '. Conversely, to decrease $d$, both a lack in luminance contrast and absolute luminance were required. For signal detection performance, these findings indicate the presence of a "deficiency window" as a function of an interaction between base luminance and contrast ratio. The identified "deficiency window" in our study was a combination of a luminance value $<0.6 \mathrm{~cd} / \mathrm{m}^{2}$ and a suboptimal luminance ratio $\left(<1.7\right.$ or $\left.C_{M}<0.24\right)$, in which we found significantly poorer performance than in all other trial combinations. It seems that if there is either an optimal luminance value or optimal contrast ratio, participants can complete the task with little decreases in performance accuracy. Also the linear regression analysis suggests that it is a power coefficient of the combined contrasts measures

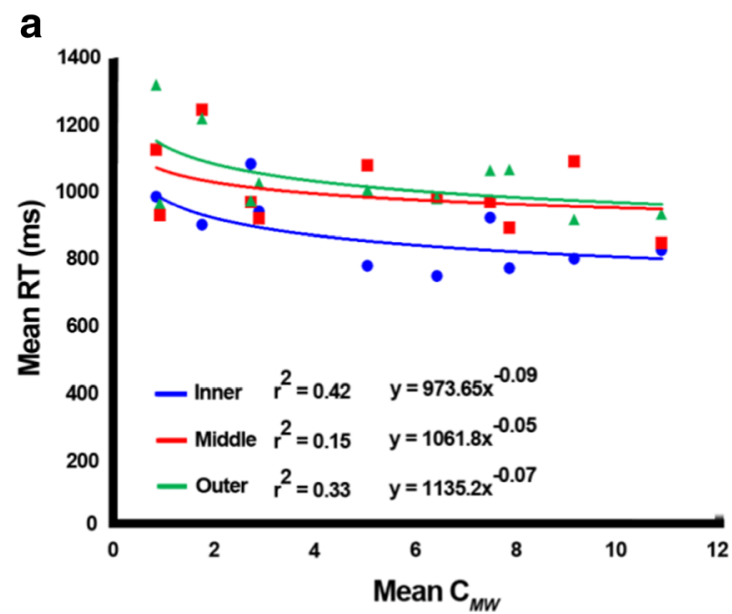

Fig. 4 RT efficiency slopes reflecting eccentricity $x$ presence interaction. Efficiency functions are presented for both absent (A) and present (B) trials. Blue lines represent the innermost stimulus trials, while Green and

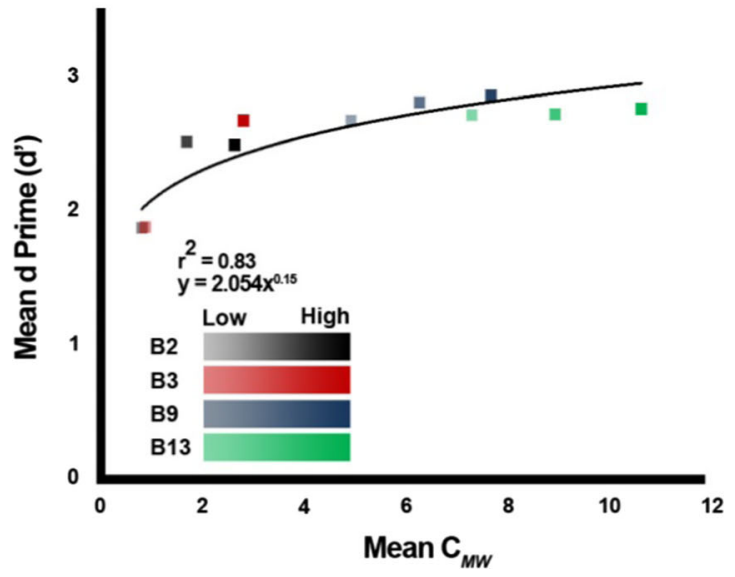

Medium and Dark $=$ High) conditions. Base colour and contrast gradients are provided within the legends for reference to each markers colour and contrast appearance. The power equation and $r^{2}$ value of the equation for each measure is plotted on the graph.

$\left(C_{M W}\right)$, which best fits the model, similar to the power functions as observed by Walkey et al. (2006a, b).

In line with our hypothesis $d$ ' was not influenced by eccentricity of the target; however, our hypothesis regarding decreased RTs as a function of increased lateralization was not supported. Eccentricity of the target only influenced RTs of the participants when the target was absent. Once the stimuli were placed at a distance greater than or equal to a distance magnitude of $6^{\circ}$ visual angle RTs were significantly slower and not faster as originally thought. When a target was present there was no significant difference between any of the stimulus positions. This result could be a function of different scenarios that will be discussed in the following.

First, presence of the target could have been sufficient to cause a pop-out characteristic and capture the participant's attention enough so that participants were able to respond quicker independent of the distance. There was effectively

\section{b}

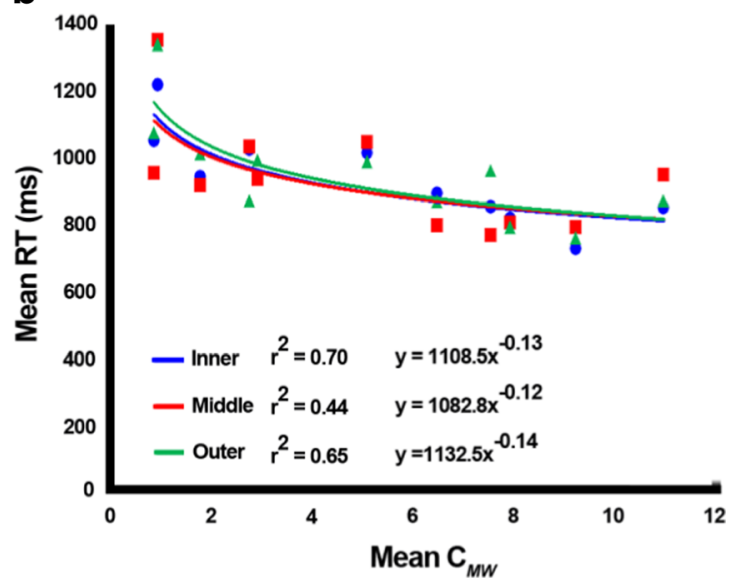

Red represent the middle and outer stimulus locations respectively. $r^{2}$ and function equations are provided on each graph. 
no difference in the target present trials with respect to the slope and power function values, whereas there was considerable difference in the slope and power function in the target absent trials. This may provide support that the target pops out or has high saliency, which normalizes the respective trial to the observed functions independent of distance, whereas a lack of a target is susceptible to decreases in performance as a function of stimulus distance.

Second, the participants might have used search patterns that could have provided a bias in either response probability or physical movement limitations. If participants were to maintain a specific search pattern throughout the experiment (i.e., left side before right), this would maintain an initial stimulus fixation on the target $50 \%$ of the time, which could have been sufficient to lower mean RT, masking any effect. Unfortunately, side of stimulus presentation was not recorded and only further testing could shed light on this phenomenon. Additionally, participants were not forced to engage solely on the center of the display, thereby forcing participants to maintain the rod maculation pertinent to the hypothesis but were instead given the liberty to adopt any completion strategy desired providing more conic stimulation on the retina. That would inherently produce a distance effect for the participants who scanned both stimuli independently, having greater distance to traverse at the more lateral stimuli locations. Unfortunately, scan patterns were not measured so that this cannot be assessed with the current data.

A third interpretation and maybe the most probable, based on the eccentricity by presence interaction, could be that with targets absent longer scan paths for more eccentric targets resulted in increased RT for these conditions but that this path effect was counteracted in the target present condition by faster processing of eccentric stimuli, resulting in no net effect of eccentricity in the target present condition. This interpretation is further supported by the results of the regression plots when considering eccentricity $\mathrm{r}^{2}$ values (independent of target presence). The inner and outer positions were effectively identical while the middle stimulus demonstrated the weakest $r^{2}$ coefficient, which may reflect a transitional "blind spot" (higher variability). Decreases observed at the 6-degree position may be a function of a lack of retinal rod stimulation (fitting our hypothesis), whereas the decreases in performance at 10 degrees may be a function of the distance needed to scan the stimuli. ${ }^{3}$

The present study should foster several follow-up experiments that may wish to address the impact of dark adaptation verification and the generalizability of the present findings to

\footnotetext{
${ }^{3}$ Due to the fact that neuronal response to a disk stimulus in a given visual receptive field can vary with increasing eccentricity, increasing stimulus size, or the combination of the two (Press, Brewer, Dougherty, Wade \& Wandell, 2001), the spatial summation occurring or the primary cortical area of activation also can be confounding results. Because there was no verification of the cortical stimulation/activation, confirmation is not possible.
}

more complex visual tasks such as visual search. Additionally, the inclusion of eye tracking would provide insight as to the scanning pattern utilized through the experiment. In that respect, an emphasis could be placed on target fixation allowing more inference relating to the temporal and spatial engagement of the receptive fields. Addition of the measurement of visual evoked potentials (VEP) could allow for verification of the cortical receptive field being activated further confirming or providing greater insight of our eccentricity results.

\section{Conclusions}

Using a simple visual discrimination task between two stimuli (one target and one distractor), which also could be considered a very simple visual search task with a set size of two, we identified a "deficiency window" under low mesopic threshold demonstrating how the manipulation of absolute luminance, luminance contrast, or the combination of both contributes to both speed and accuracy within the paradigm. For the first time, our study presents behavioural performance functions observed within this range and provides a solid foundation from both a paradigm and analysis perspective to further elucidate the mechanisms of visual perception affecting performance in low mesopic and potentially complete scotopic environments.

Acknowledgments The authors acknowledge funding support from the German Academic Exchange Service (DAAD) and the Cognitive Systems and Processes (COSYP) research center at Jacobs University Bremen. We thank Dr. Claudia Voelcker-Rehage and Dr. Andreas Birk for fruitful comments on the study design.

\section{References}

Akashi, Y., \& Rea, M. (2002). Peripheral detection while driving under a mesopic light level. Journal of the Illuminating Engineering Society, 31(1), 85-94. doi:10.1080/00994480.2002.10748374

Alferdinck, J. W. A. M. (2006). Target detection and driving behaviour measurements in a driving simulator at mesopic light levels. Ophthalmic and Physiological Optics, 26(3), 264-280. doi:10.1111/j.1475-1313.2006.00324.x

Anstis, S. (2002). The purkinje rod-cone shift as a function of luminance and retinal eccentricity. Vision Research, 42(22), 2485-2491.

Blackwell, H. R. (1946). Contrast thresholds of the human eye. Journal of the Optical Society of America, 36(11), 624-643.

Braithwaite, J. J., Watson, D. G., Andrews, L., \& Humphreys, G. W. (2010). Visual search at isoluminance: Evidence for enhanced color weighting in standard sub-set and preview-based visual search. Vision Research, 50(14), 1414-1425. doi:10.1016/j.visres.2010.01.013

Eloholma, M., Ketomäki, J., Orreveteläinen, P., \& Halonen, L. (2006). Visual performance in night-time driving conditions. Ophthalmic and Physiological Optics, 26(3), 254-263. doi:10.1111/j.14751313.2006.00313.x

Gloriani, A. H., Matesanz, B. M., Barrionuevo, P. A., Arranz, I., Issolio, L., Mar, S., \& Aparicio, J. A. (2016). Influence of background size, 
luminance and eccentricity on different adaptation mechanisms. Vision Research, 125, 12-22. doi:10.1016/j.visres.2016.04.008

Hadjikhani, N., \& Tootell, R. B. (2000). Projection of rods and cones within human visual cortex. Human Brain Mapping, 9(1), 55-63. doi:10.1002/(SICI)1097-0193(2000)9:1<55::AID-HBM6>3.0. $\mathrm{CO} ; 2-\mathrm{U}$

Hertenstein, H., Bach, M., Gross, N. J., \& Beisse, F. (2016). Marked dissociation of photopic and mesopic contrast sensitivity even in normal observers. Graefe's Archive for Clinical and Experimental Ophthalmology = Albrecht Von Graefes Archiv Fur Klinische Und Experimentelle Ophthalmologie, 254(2), 373-384. doi:10.1007 /s00417-015-3020-4

Hiraoka, T., Hoshi, S., Okamoto, Y., Okamoto, F., \& Oshika, T. (2015). Mesopic functional visual acuity in normal subjects. PloS One, 10(7), e0134505. doi:10.1371/journal.pone.0134505

Koefoed, V. F., Baste, V., Roumes, C., \& Hovding, G. (2015). Contrast sensitivity measured by two different test methods in healthy, young adults with normal visual acuity. Acta Ophthalmologica, 93(2), 154-161. doi:10.1111/aos.12487

Kunar, M. A., \& Watson, D. G. (2011). Visual search in a multi-element asynchronous dynamic (MAD) world. Journal of Experimental Psychology.Human Perception and Performance, 37(4), 1017 1031. doi:10.1037/a0023093

Lee, H., Legge, G. E., \& Ortiz, A. (2003). Is word recognition different in central and peripheral vision? Vision Research, 43(26), 2837-2846. doi:10.1016/S0042-6989(03)00479-6

Macmillan, N., \& Creelman, C. (2005). The yes-no experiment: Sensitivity. Detection theory: A user's guide (2nd ed., pp. 8). New Jersey: Lawrence Erlbaum Associates.

Michelson, A. A. (1927). Studies in optics. Chicago, Ill.: The University of Chicago Press.

Peli, E. (1990). Contrast in complex images. Journal of the Optical Society of America.A, Optics and Image Science, 7(10), 2032-2040.

Perez, M. J., Puell, M. C., Sanchez, C., \& Langa, A. (2003). Effect of a yellow filter on mesopic contrast perception and differential light sensitivity in the visual field. Ophthalmic Research, 35(1), 54-59.

Plainis, S., \& Murray, I. J. (2000). Neurophysiological interpretation of human visual reaction times: Effect of contrast, spatial frequency and luminance. Neuropsychologia, 38(12), 1555-1564.

Plainis, S., \& Murray, I. J. (2005). Magnocellular channel subserves the human contrast-sensitivity function. Perception, 34(8), 933-940.

Plainis, S., Murray, I. J., \& Charman, W. N. (2005). The role of retinal adaptation in night driving. Optometry and Vision Science : Official
Publication of the American Academy of Optometry, 82(8), 682688.

Press, W. A., Brewer, A. A., Dougherty, R. F., Wade, A. R., \& Wandell, B. A. (2001). Visual areas and spatial summation in human visual cortex. Vision Research, 41(10-11), 1321-1332.

Rauschenberger, R. (2003). When something old becomes something new: Spatiotemporal object continuity and attentional capture. Journal of Experimental Psychology.Human Perception and Performance, 29(3), 600-615.

Rea, M. S., \& Bullough, J. D. (2007). Making the move to a unified system of photometry. Lighting Research and Technology, 39(4), 393-408. doi:10.1177/1477153507077395

Riggs, L. (1965). Visual acuity. In C. Graham (Ed.), Vision and visual perception (pp. 321-349). New York: Wiley.

Skottun, B. C. (2013). On using isoluminant stimuli to separate magnoand parvocellular responses in psychophysical experiments-a few words of caution. Behavior Research Methods, 45(3), 637-645. doi:10.3758/s13428-012-0290-1

Skottun, B. C., \& Skoyles, J. R. (2008). Visual search: Magno- and parvocellular systems or color and luminance processes? The International Journal of Neuroscience, 118(9), 1259-1267. doi:10.1080/00207450701239434

Szalmas, A., Bodrogi, P., \& Sik-Lanyi, C. (2006). Characterizing luminous efficiency functions for a simulated mesopic night driving task based on reaction time. Ophthalmic and Physiological Optics, 26(3), 281-287. doi:10.1111/j.1475-1313.2006.00406.x

Wald, G. (1945). Human vision and the spectrum. Science, 101(2635), 653.

Walkey, H. C., Harlow, J. A., \& Barbur, J. L. (2006a). Changes in reaction time and search time with background luminance in the mesopic range. Ophthalmic \& Physiological Optics : The Journal of the British College of Ophthalmic Opticians (Optometrists), 26(3), 288-299.

Walkey, H. C., Harlow, J. A., \& Barbur, J. L. (2006b). Characterising mesopic spectral sensitivity from reaction times. Vision Research, 46(25), 4232-4243.

Watson, D. G., \& Humphreys, G. W. (2002). Visual marking and visual change. Journal of Experimental Psychology. Human Perception and Performance, 28(2), 379-395.

Yoshimoto, S., Okajima, K., \& Takeuchi, T. (2016). Motion perception under mesopic vision. Journal of Vision, 16(1), 16. doi:10.1167/16.1.16

Zele, A. J., \& Cao, D. (2015). Vision under mesopic and scotopic illumination. Frontiers in Psychology, 5, 1594. doi:10.3389/fpsyg.2014.01594 